\title{
Universal scalings of universal scaling exponents
}

\author{
Rafael de la Llave ${ }^{1}$, Arturo Olvera ${ }^{2}$, Nikola P. Petrov ${ }^{3}$ \\ ${ }^{1}$ Department of Mathematics, University of Texas, Austin, TX 78712 \\ 2 IIMAS-UNAM, FENOMEC, Apdo. Postal 20-726, México D. F. 01000, Mexico \\ ${ }^{3}$ Department of Mathematics, University of Oklahoma, Norman, OK 73019 \\ E-mail: llave@math.utexas.edu, aoc@mym.iimas.unam.mx, npetrov@math.ou.edu
}

\begin{abstract}
In the last decades, renormalization group (RG) ideas have been applied to describe universal properties of different routes to chaos (quasi-periodic, period doubling or tripling, Siegel disk boundaries, etc.). Each of the RG theories leads to universal scaling exponents which are related to the action of certain RG operators.

The goal of this announcement is to show that there is a principle that organizes many of these scaling exponents. We give numerical evidence that the exponents of different routes to chaos satisfy approximately some arithmetic relations. These relations are determined by combinatorial properties of the route and become exact in an appropriate limit.
\end{abstract}

PACS numbers: 05.45.Df 05.10.-a 05.10.Cc 


\section{Introduction}

One of the most striking discoveries in dynamical systems in the last decades has been the existence of scaling relations and self-similarity in the transition to chaotic behavior. By now there is a plethora of transitions each with its own exponents. Many scaling relations have been explained by renormalization group ( $R G)$ theory [1]. Our goal here is to report on some organization among the scaling exponents of different transitions.

In many transitions, one can associate a combinatorics to the maps. For example, in unimodal maps we can prescribe the kneading sequence or in quasiperiodic maps the rotation number. There are natural operations among these combinatirics. For example, one can use the $*$-operation among finite kneading sequences [2] or just the juxtaposition among finite continued fraction expansions.

If we fix one of these combinatorics, we can consider successions of bifurcations obtained by selecting mappings whose combinatorics is a power of the operation. For example, the standard period doubling correspondes to considering $(R *)^{n}$ and the usual quasi-periodic route for the golden mean to take continued fractions $F_{n} / F_{n+1}=$ $[1, \ldots, 1]$. If we choose other sequence to raise to increasing powers, we obtain different routes to chaos, which are often found to have scaling properties.

In this short paper we formulate the Principle of Approximate Combination of Scaling Exponents (PACSE for short). PACSE asserts that the scaling exponents of different transitions are related. PACSE can be formulated briefly as follows.

(A) If the combinatorics of two routes to chaos are joined, by the natural operation, the scaling exponents of the joint combinatorics are approximated by the product of the exponents of the two original routes.

(B) The approximate product rule in (A) becomes exact if the combination is repeated infinitely many times.

(C) The convergence in part (B) is exponential.

In Sections 2-4, we make explicit the meaning of the combinatorics and their combination rules in some examples. We also present numerical evidence for the points (A), (B), (C) above. In Section 5 we indicate some theoretical ideas why PACSE should be true.

\section{PACSE for critical circle maps}

\subsection{Real- and parameter-space scaling exponents}

We first describe the principle of approximate combination in the case of quasiperiodic route to chaos.

In $[3,4,5,6]$ there is a description of phenomena in families of maps with a cubic critical point and rotation number equal to the golden mean, $\sigma_{\mathrm{G}}:=\frac{1}{2}(\sqrt{5}-1)$.

In this paper we consider more general quasi-periodic transitions. The different quasi-periodic transitions are characterized by an irrational number $\rho \in[0,1)$ called 
the rotation number. The combinatorics of the transition is given by the continued fraction expansion (CFE) of $\rho[7]$. We write $\left\langle a_{1}, a_{2}, \ldots\right\rangle=1 /\left(a_{1}+1 /\left(a_{2}+\cdots\right)\right)$. We consider numbers whose $\mathrm{CEF}$ is eventually periodic. Given finite sequences of natural numbers $\mathrm{A}=\left(a_{1}, a_{2}, \ldots, a_{p}\right)$ and $\mathrm{B}=\left(b_{1}, b_{2}, \ldots, b_{q}\right)$, we define their concatenation $\mathrm{AB}=\left(a_{1}, a_{2}, \ldots, a_{p}, b_{1}, b_{2}, \ldots, b_{q}\right)$ and denote repeated concatenations by by exponents.

We will denote by $\left\langle A B^{\infty}\right\rangle=\langle A B B \ldots\rangle$ the irrational number whose CFE is the concatenation of $A$ and infinitely many copies of $B$. To such a number, we can associate a sequence of rational numbers which converge to it, $\frac{P_{n}}{Q_{n}}=\left\langle\mathrm{AB}^{n}\right\rangle$.

As prototypes of circle maps we consider the two-parameter family

$$
f_{\omega, \beta}(x)=[x+\omega+\beta g(x)] \bmod 1,
$$

where $g(x)$ is a smooth periodic function of period 1, i.e., $\sin 2 \pi x$. If $f^{\prime}(x)$ becomes 0 at one point $c$ (and, therefore, $f^{-1}$ is not differentiable), we say that $f$ is a critical map and call $c$ the critical point of $f$. Below $f^{n}(x)$ will stand for the $n$th iteration, i.e., to the map $f$ applied $n$ times: $f^{n}(x)=f \circ f \circ \cdots \circ f(x)$.

For many rotation numbers of the form $\left\langle A B^{\infty}\right\rangle$ the following behavior has been observed.

(a) Parameter-space scaling. For a fixed value of the nonlinearity parameter $\beta$ in $(1)$, let $I_{n}(\beta)$ be a phase locking interval, i.e., the interval of values of the parameter

$\omega$ for which the circle map $f_{\omega, \beta}$ has rational rotation number $\frac{P_{n}}{Q_{n}}=\left\langle\mathrm{AB}^{n}\right\rangle$. Then the lengths of the phase locking intervals behave with $n$ as

$$
\left|I_{n}(\beta)\right| \approx C \delta_{\mathrm{B}}^{-n}
$$

where $\delta_{\mathrm{B}}$ is a universal number, that is, a number that depends only on $\mathrm{B}$ (but not on the head $A$ of $\left.C F E\left\langle A B^{\infty}\right\rangle\right)$ and on the order of the critical point $c$, but is otherwise independent of the families $f_{\omega, \beta}$ (when the families range over a small enough neighborhood). We will indicate the order of the critical point $c$ with a superscript on $\delta_{\mathrm{B}}$.

(b) Real-space scaling (scaling of recurrences). Let $f$ be a critical circle map with critical point $c$, and rotation number $\left\langle\mathrm{AB}^{\infty}\right\rangle$. Then the iterates $f^{Q_{n}}(c)$ approach $c$ geometrically:

$$
\left|f^{Q_{n}}(c)-c\right| \approx C \alpha_{\mathrm{B}}^{-n}
$$

where $\alpha_{\mathrm{B}}$ is a universal number (in the same sense as for $\delta_{\mathrm{B}}$ ). A superscript will denote the order of criticality.

\subsection{Formulation of PACSE for critical circle maps}

For circle maps, PACSE is expressed as follows.

(i) For a fixed order of criticality of $c$, there exist constants $C_{1}$ and $C_{2}$ such that

$$
C_{1} \leq \frac{\delta_{\mathrm{AB}}}{\delta_{\mathrm{A}} \delta_{\mathrm{B}}} \leq C_{2}, \quad C_{1} \leq \frac{\alpha_{\mathrm{AB}}}{\alpha_{\mathrm{A}} \alpha_{\mathrm{B}}} \leq C_{2}
$$


where $C_{1}$ and $C_{2}$ depend only on $\max \left(a_{1}, \ldots, a_{p}, b_{1}, \ldots, b_{q}\right)$.

(ii) For a fixed order of criticality of $c$ and fixed $A$ and $B$, the following limits exist:

$$
\lim _{k \rightarrow \infty} \frac{\delta_{\mathrm{A}^{k} \mathrm{~B}}}{\left(\delta_{\mathrm{A}}\right)^{k} \delta_{\mathrm{B}}}, \quad \lim _{k \rightarrow \infty} \frac{\alpha_{\mathrm{A}^{k} \mathrm{~B}}}{\left(\alpha_{\mathrm{A}}\right)^{k} \alpha_{\mathrm{B}}} .
$$

(iii) For a fixed order of criticality of $c$ and fixed $\mathrm{A}$ and $\mathrm{B}$, the ratios $\mathscr{D}_{k}=\frac{\delta_{\mathrm{A}} k_{\mathrm{B}}}{\left(\delta_{\mathrm{A}}\right)^{k} \delta_{\mathrm{B}}}$ and $\mathscr{A}_{k}=\frac{\alpha_{\mathrm{A}} k_{\mathrm{B}}}{\left(\alpha_{\mathrm{A}}\right)^{k} \alpha_{\mathrm{B}}}$ approach their limiting values $\mathscr{D}_{\infty}$ and $\mathscr{A}_{\infty}$ exponentially:

$$
\left|\mathscr{D}_{k}-\mathscr{D}_{\infty}\right| \approx C \xi^{k}, \quad\left|\mathscr{A}_{k}-\mathscr{A}_{\infty}\right| \approx C \eta^{k}
$$

for some constants $\xi$ and $\eta$.

The bounds $(2)$ are quite surprising because $\alpha_{\mathrm{A}}$ and especially $\delta_{\mathrm{A}}$ are huge when the length of $A$ is large.

\subsection{Evidence for PACSE for critical circle maps}

We studied the following families of circle maps.

- The "standard" cubic critical (C) family $\left(0 \leq K<\frac{4}{3}\right)$

$$
f_{K, \omega}^{\mathrm{C}}(x)=\left[x+\omega-\frac{1}{2 \pi}\left(K \sin 2 \pi x+\frac{1-K}{2} \sin 4 \pi x\right)\right] \bmod 1,
$$

where the coefficients are chosen in such a way that for every $K$ in the interval $\left(0, \frac{4}{3}\right)$, $f_{K, \omega}^{\mathrm{C}}(x)=\omega+\frac{2 \pi^{2}(4-3 K)}{3} x^{3}+\mathcal{O}\left(x^{5}\right)$.

- The "standard" quintic critical (Q) family $\left(\frac{1}{2} \leq K<\frac{3}{2}\right)$

$$
f_{K, \omega}^{\mathrm{Q}}(x)=\left[x+\omega-\frac{1}{2 \pi}\left(K \sin 2 \pi x+\frac{9-8 K}{10} \sin 4 \pi x+\frac{3 K-4}{15} \sin 6 \pi x\right)\right] \bmod 1,
$$

where the coefficients are chosen in such a way that for every $K$ in the interval $\left(\frac{1}{2}, \frac{3}{2}\right)$, $f_{K, \omega}^{\mathrm{Q}}(x)=\omega+\frac{8 \pi^{4}(3-2 K)}{5} x^{5}+\mathcal{O}\left(x^{7}\right)$.

- The maps

$$
f(x)=x+\omega-\frac{b}{2 \pi} \frac{\sin 2 \pi x}{a-\cos 2 \pi x}
$$

with $(a, b)=(2,1)$, for which $f$ is cubic critical $\left[f(x)=\omega+\frac{8}{3} \pi^{2} x^{3}+\mathcal{O}\left(x^{5}\right)\right]$; and $(a, b)=(-2,-3)$, for which $f$ is quintic critical $\left[f(x)=\omega+\frac{4}{45} \pi^{4} x^{5}+\mathcal{O}\left(x^{7}\right)\right]$.

Since the maps (5) with a fixed order of critical point do not contain any free parameter, we only computed the $\alpha$ exponents. This was to reassure us that the results apply to functions with infinitely harmonics.

For more detail on algorithms for computing the critical values, we refer to [8], which only considered the case of the golden mean. Table 1 presents our numerical results for the scaling exponents $\delta$ and $\alpha$ for the cases of cubic and quintic critical maps. In the quintic critical case we studied rotation numbers of the form $\left\langle\left(1^{k} 2\right)^{\infty}\right\rangle$, while in the cubic critical case we did it for $\left\langle\left(1^{k} 2\right)^{\infty}\right\rangle$ and $\left\langle\left(1^{k} 3\right)^{\infty}\right\rangle$. The errors do not exceed 2 in the last digit. When we did not detect good enough convergence we just put "?".

In Table 2 we present the ratios in (2) and (3), keeping only the number of digits 
Table 1. Scaling exponents of $\mathrm{C}$ and $\mathrm{Q}$ maps with rotation numbers $\left\langle\mathrm{B}^{\infty}\right\rangle, \mathrm{B}=\left(1^{k} 2\right)$, and of $\mathrm{C}$ maps with rotation numbers $\left\langle\mathrm{B}^{\infty}\right\rangle, \mathrm{B}=\left(1^{k} 3\right)$.

\begin{tabular}{|c|c|c|c|c|c|c|}
\hline \multirow[b]{2}{*}{$k$} & \multicolumn{2}{|c|}{ Cubic critical, $\mathrm{B}=\left(1^{k} 2\right)$} & \multicolumn{2}{|c|}{ Cubic critical, $\mathrm{B}=\left(1^{k} 3\right)$} & \multicolumn{2}{|c|}{ Quintic critical, $\mathrm{B}=\left(1^{k} 2\right)$} \\
\hline & $\delta_{1^{k} 2}^{\mathrm{C}}$ & $\alpha_{1^{k} 2}^{\mathrm{C}}$ & $\delta_{1^{k} 3}^{\mathrm{C}}$ & $\alpha_{1^{k} 3}^{\mathrm{C}}$ & $\delta_{1^{k} 2}^{\mathrm{Q}}$ & $\alpha_{1^{k} 2}^{\mathrm{Q}}$ \\
\hline 0 & 6.79922516 & 1.58682670 & 13.760284 & 1.855060 & 7.7912246 & 1.3791501 \\
\hline 1 & 17.66905276 & 1.9691355 & 31.623877 & 2.17411 & 21.573320 & 1.5985 \\
\hline 2 & 52.04449 & 2.590589 & 98.32467 & 2.945324 & 68.620816 & 1.9392 \\
\hline 3 & 145.425152 & 3.308635 & 269.104 & 3.71001 & 205.43 & 2.2997 \\
\hline 4 & 414.51561 & 4.28301 & 774.04 & 4.836423 & 629.5 & 2.7536 \\
\hline 5 & 1171.7123 & 5.5067 & 2179.3 & 6.19630 & 1910.6 & 3.2836 \\
\hline 6 & 3323.73 & 7.1039 & 6193 & 8.0082 & 5820 & 3.9216 \\
\hline 7 & 9413.7 & 9.14860 & 17530 & 10.3035 & 17710 & 4.6815 \\
\hline 8 & 26681 & 11.7923 & $49700_{ \pm 20}$ & 13.287 & 53500 & 5.590 \\
\hline 9 & 75590 & 15.1929 & 140800 & 17.117 & 160000 & 6.677 \\
\hline 10 & 214000 & 19.579 & $400000_{ \pm 20000}$ & 22.061 & 500000 & 7.970 \\
\hline 11 & 607900 & 25.230 & $?$ & 28.428 & 1600000 & 9.53 \\
\hline
\end{tabular}

Table 2. Ratios of scaling exponents as in (2) for the data from Table 1.

\begin{tabular}{|c|c|c|c|c|c|c|}
\hline \multirow[b]{2}{*}{$k$} & \multicolumn{2}{|c|}{ Cubic critical, $\mathrm{B}=\left(1^{k} 2\right)$} & \multicolumn{2}{|c|}{ Cubic critical, $\mathrm{B}=\left(1^{k} 3\right)$} & \multicolumn{2}{|c|}{ Quintic critical, $\mathrm{B}=\left(1^{k} 2\right)$} \\
\hline & $\frac{\delta_{1^{k} 2}^{\mathrm{C}}}{\left(\delta_{1}^{\mathrm{C}}\right)^{k} \delta_{2}^{\mathrm{C}}}$ & $\frac{\alpha_{1^{k} 2}^{\mathrm{C}}}{\left(\alpha_{1}^{\mathrm{C}}\right)^{k} \alpha_{2}^{\mathrm{C}}}$ & $\frac{\delta_{1^{k} 3}^{\mathrm{C}}}{\left(\delta_{1}^{\mathrm{C}}\right)^{k} \delta_{3}^{\mathrm{C}}}$ & $\frac{\alpha_{1^{k} 3}^{\mathrm{C}}}{\left(\alpha_{1}^{\mathrm{C}}\right)^{k} \alpha_{3}^{\mathrm{C}}}$ & $\frac{\delta_{1^{k} 2}^{\mathrm{Q}}}{\left(\delta_{1}^{\mathrm{Q}}\right)^{k} \delta_{2}^{\mathrm{Q}}}$ & $\frac{\alpha_{1^{k} 2}^{\mathrm{Q}}}{\left(\alpha_{1}^{\mathrm{Q}}\right)^{k} \alpha_{2}^{\mathrm{Q}}}$ \\
\hline 1 & 0.9170936095 & 0.96302277 & 0.81104983 & 0.909524 & 0.90981984 & 0.97084 \\
\hline 2 & 0.9533118 & 0.9832182 & 0.8899277 & 0.9562160 & 0.95091785 & 0.98652 \\
\hline 3 & 0.940068655 & 0.9745199 & 0.859552 & 0.934735 & 0.93539 & 0.97995 \\
\hline 4 & 0.94562895 & 0.978997 & 0.87252 & 0.9456449 & 0.9418 & 0.98283 \\
\hline 5 & 0.94332356 & 0.97682 & 0.86694 & 0.940214 & 0.93926 & 0.98170 \\
\hline 6 & 0.944333 & 0.97793 & 0.8694 & 0.94302 & 0.9401 & 0.98206 \\
\hline 7 & 0.94389 & 0.977369 & 0.8685 & 0.941586 & 0.9300 & 0.98199 \\
\hline 8 & 0.94411 & 0.977671 & 0.869 & 0.94231 & 0.924 & 0.9822 \\
\hline 9 & 0.9439 & 0.977519 & 0.869 & 0.94207 & 0.93 & 0.9827 \\
\hline 10 & 0.943 & 0.97761 & 0.87 & 0.94226 & 0.94 & 0.9825 \\
\hline 11 & 0.945 & 0.97765 & $?$ & 0.94229 & 1.0 & 0.984 \\
\hline
\end{tabular}

such that the error does not exceed 2 in the last digit, unless otherwise specified. In the computations we used the values of the scaling exponents $\delta_{1}$ and $\alpha_{1}$ for rotation number golden mean, $\left\langle 1^{\infty}\right\rangle: \delta_{1}^{\mathrm{C}}=2.8336106559, \alpha_{1}^{\mathrm{C}}=1.28857456, \delta_{1}^{\mathrm{Q}}=3.04337774$, $\alpha_{1}^{\mathrm{Q}}=1.193857$.

As a numerical confirmation of the exponential convergence of the ratios $\mathscr{D}_{k}$ and $\mathscr{A}_{k}$ (see (4)), we present in Figure 1 the differences $\left|\mathscr{D}_{k}-\mathscr{D}_{\infty}\right|$ and $\left|\mathscr{A}_{k}-\mathscr{A}_{\infty}\right|$ versus $k$. Note that for large values of $k$ the computation is very sensitive to numerical errors.

We also studied numerically the scaling exponents for maps with rotation numbers that are eventually periodic (like $\left\langle 31^{\infty}\right\rangle,\left\langle 3^{2} 1^{\infty}\right\rangle,\left\langle 3^{2}(12)^{\infty}\right\rangle$ ), and found that the values of the scaling exponents depend only on the tail of the continued fraction expansions, 

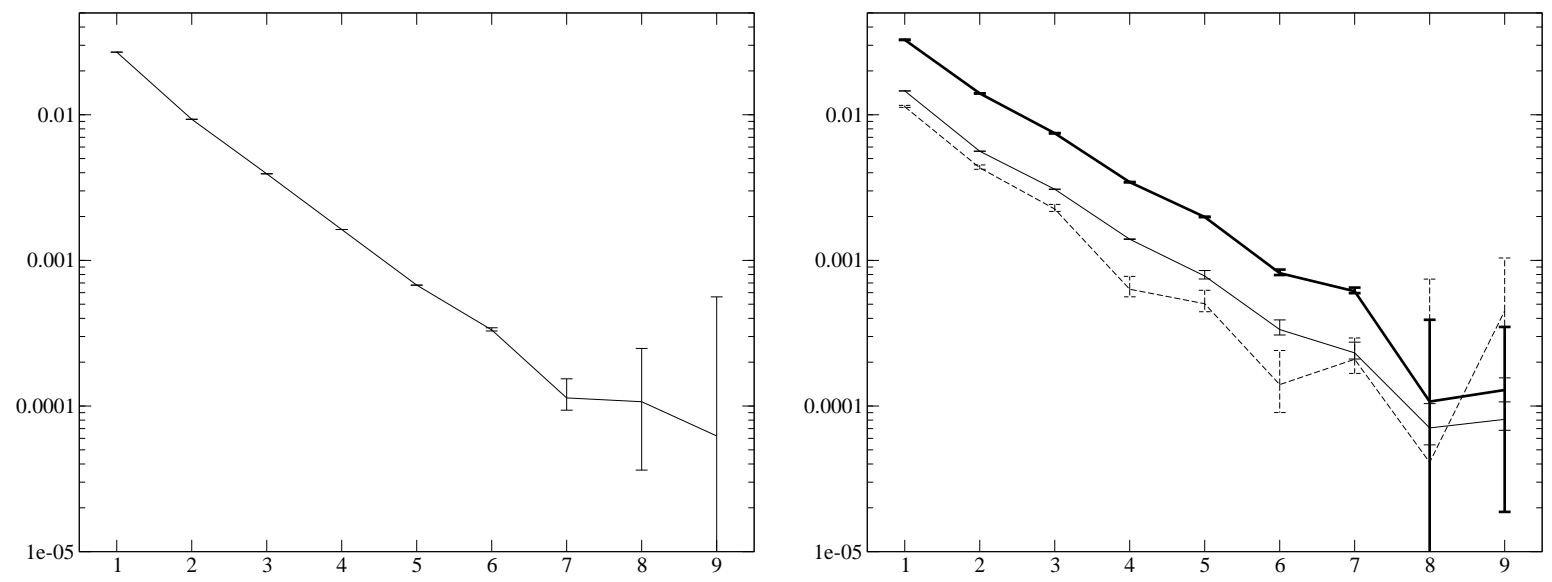

Figure 1. Left: log-linear plot of the differences $\left|\delta_{1^{k} 2}^{\mathrm{C}} /\left[\left(\delta_{1}^{\mathrm{C}}\right)^{k} \delta_{2}^{\mathrm{C}}\right]-0.944\right|$; right: log-linear plot of the differences $\mid\left[\alpha_{1^{k} 2}^{\mathrm{C}} /\left[\left(\alpha_{1}^{\mathrm{C}}\right)^{k} \alpha_{2}^{\mathrm{C}}\right]-0.9776 \mid\right.$ (thin lines), $\left|\left[\alpha_{1^{k} 3}^{\mathrm{C}} /\left(\alpha_{1}^{\mathrm{C}}\right)^{k} \alpha_{3}^{\mathrm{C}}\right]-0.9422\right|$ (thick lines), $\left|\left[\alpha_{1^{k} 2}^{\mathrm{Q}} /\left(\alpha_{1}^{\mathrm{Q}}\right)^{k} \alpha_{2}^{\mathrm{Q}}\right]-0.9822\right|$ (dashed lines) vs. $k$ for $k=1,2, \ldots, 9$.

as predicted by the general theory.

\section{PACSE for area-preserving twist maps of the cylinder}

We considered the standard (Taylor-Chirikov) family of area-preserving twist maps,

$$
\left(x^{\prime}, y^{\prime}\right)=\left(x+y^{\prime}, y+\frac{K}{2 \pi} \sin 2 \pi x\right) .
$$

We refer to [9] for background on the standard maps and the algorithms used, although [9] studies only one fixed rotation number. RG theory of such maps is developed in $[10,6,11,12]$.

Given a rotation number $\left\langle\mathrm{B}^{\infty}\right\rangle$, the critical events we studied are the existence of tangencies between the stable and unstable manifolds of periodic orbits with rotation number $\left\langle\mathrm{B}^{n}\right\rangle$ and $\left\langle\mathrm{B}^{n+1}\right\rangle$. Denoting the critical parameter values by $K_{n}$, and the area of the lobes enclosed by the tangency by $L_{n}$, we observed the following scalings:

$$
\left|K_{n}-K_{\infty}\right| \approx C \Delta_{\mathrm{B}}^{-n}, \quad L_{n} \approx C \lambda_{\mathrm{B}}^{-n},
$$

where $\Delta_{B}$ and $\lambda_{B}$ are universal numbers depending only on B. In Table 3 we summarize our numerical results.

\section{Other contexts for PACSE}

In this section, we describe some other contexts for PACSE, making precise the meaning

of combinatorics. We have some numerical data, but will not present it to keep the announcement short. 
Table 3. Scaling exponents of critical area-preserving twist maps with rotation numbers $\left\langle\left(21^{k}\right)^{\infty}\right\rangle$; we used that $\Delta_{1}=1.62802, \Delta_{2}=2.4569, \lambda_{1}=4.339143$, $\lambda_{2}=14.60$.

\begin{tabular}{lrcll}
\hline$k$ & $\Delta_{21^{k}}$ & $\lambda_{21^{k}}$ & $\frac{\Delta_{21^{k}}}{\Delta_{2}\left(\Delta_{1}\right)^{k}}$ & $\frac{\lambda_{21^{k}}}{\lambda_{2}\left(\lambda_{1}\right)^{k}}$ \\
\hline 1 & 3.778 & 59.548 & 0.944 & 0.939 \\
2 & 6.298 & 284.53 & 0.967 & 1.035 \\
3 & 10.168 & 1141.44 & 0.959 & 0.956 \\
\hline
\end{tabular}

\subsection{Unimodal maps of the interval}

Renormalization for unimodal maps is analogous to the renormalization for circle maps. The kneading sequences play a role similar to the role of the rotation numbers. An analogue of concatenation of continued fractions is the $*$-operation of $[13,14,2,15]$. For a given a kneading sequence $\mathrm{K}$ and a family $f_{\lambda}$, it is standard to check for the parameters $\lambda_{n}$ characterized by the critical point being periodic and having an itinerary given by $\mathrm{K}^{* n}$. In this case, one can obtain parameter- and real-space scaling exponents by

$$
\left|\lambda_{n}-\lambda_{\infty}\right| \approx C \delta_{\mathrm{K}}^{-n}, \quad f_{\lambda_{n+1}}^{\left|\mathrm{K}^{* n}\right|}(0) \approx C \alpha_{\mathrm{K}}^{-n} .
$$

Again, $\delta_{\mathrm{K}}, \alpha_{\mathrm{K}}$ are universal numbers which depend on $\mathrm{K}$. If $\mathrm{K}=\mathrm{K}_{1} * \mathrm{~K}_{2}$, PACSE predicts that

$$
\delta_{\mathrm{K}} \approx \delta_{\mathrm{K}_{1}} \delta_{\mathrm{K}_{2}}, \quad \alpha_{\mathrm{K}} \approx \alpha_{\mathrm{K}_{1}} \alpha_{\mathrm{K}_{2}}
$$

\subsection{Boundaries of Siegel disks}

We recall that Siegel disks are the domains of stability around the origin of maps of the complex plane of the form $f(z)=a z+O\left(z^{2}\right)$ where $a=\exp \left(2 \pi i\left\langle\mathrm{~B}^{\infty}\right\rangle\right)$.

Siegel disks have been intensively studied from the renormalization point of view since $[16,17]$. It has been found that, in many cases, the boundaries of the Siegel disks contain a critical point $c$. The renormalization theories are very similar to that of rotations since multiplication by $a$ is just a rotation, so that the combinatorics are the same as those of the circle maps. We can define real-space scaling exponents by $f^{Q_{n}}(c)-c \approx C \alpha_{\mathrm{B}}^{-n}$ and parameter-space scaling by searching for $a_{n}$ such that $f^{Q_{n}}(c)=c$ and verifying $a_{n}-a \approx C \delta_{\mathrm{B}}^{-n}$, where again $\alpha_{\mathrm{B}}$ and $\delta_{\mathrm{B}}$ are universal but depend on B. In contrast with the other cases mentioned above, the scaling exponents are complex numbers. Nevertheless, for each level of renormalization, the scaling exponents conjugate.

We have that

$$
\left|\delta_{\mathrm{AB}}\right| \approx\left|\delta_{\mathrm{A}}\right|\left|\delta_{\mathrm{B}}\right|, \quad\left|\alpha_{\mathrm{AB}}\right| \approx\left|\alpha_{\mathrm{A}}\right|\left|\alpha_{\mathrm{B}}\right| .
$$


4.3. Rigid rotations and smooth diffeomorphisms of the circle

For rigid rotations of the circle PACSE follows from a detailed study [18] of the Gauss map [7]. For circle diffeomorphisms PACSE for real-space scalings follows from the fact that the map can be smoothly conjugated to a rigid rotation $[19,20]$.

\section{4. p-renormalization}

In [21], the authors considered some special kneading sequences $\mathrm{K}=R L L L \ldots L$, for which they could compute rigorously the asymptotics of the scaling exponents. By extending slightly their computation, one can verify PACSE both for parameter- and real-space scaling [18].

\subsection{Unimodal maps that are functions of $|x|^{1+\epsilon}$}

The papers $[22,14]$ consider unimodal maps that are functions of $|x|^{1+\epsilon}$ and construct fixed points of renormalization. They present calculations of some fixed points and their scaling exponents for $\epsilon$ small. By extending their calculations slightly, one can verify [18] the first statement of PACSE up to leading order in $\epsilon$. Indeed, some version of PACSE for parameter-space scaling is mentioned in passing in [14, p. 276].

\section{Conclusions}

We established numerically some approximate relations between several scaling exponents of different RGs.

The existence of this regularity seems to be good evidence that there is a global RG applicable for maps of all rotations (or all kneading sequences). It seems possible that PACSE can be considered evidence for certain dynamical behaviors of these global renormalization operators. The paper [18] suggests that PACSE is evidence for the existence of a horseshoe with 1-dimensional unstable manifolds which, furthermore, satisfy some transversality conditions.

Global renormalizations have been proposed for Siegel discs [23, 24, 25, 26], unimodal maps $[27,28]$, critical circle maps [29, 30]. We hope that this paper can serve as a stimulus for the development of these theories.

\section{Acknowledgments}

The work of RdlL and NPP was partially supported by NSF grants.

\section{References}

[1] Annick Lesne. Renormalization Methods. John Wiley \& Sons, Chichester, 1998.

[2] P. Collet and J.-P. Eckmann. Iterated Maps on the Interval as Dynamical Systems. Birkhäuser, Boston, 1980. 
[3] S. J. Shenker. Scaling behavior in a map of a circle onto itself: empirical results. Phys. D, 5(2-3):405-411, 1982.

[4] S. Ostlund, D. Rand, J. Sethna, and E. D. Siggia. Universal properties of the transition from quasiperiodicity to chaos in dissipative systems. Phys. D, 8(3):303-342, 1983.

[5] M. J. Feigenbaum, L. P. Kadanoff, and S. J. Shenker. Quasiperiodicity in dissipative systems: a renormalization group analysis. Phys. D, 5(2-3):370-386, 1982.

[6] R. S. MacKay. A renormalisation approach to invariant circles in area-preserving maps. Phys. D, 7(1-3):283-300, 1983.

[7] A. Ya. Khinchin. Continued Fractions. Dover, 1997.

[8] R. de la Llave and N. P. Petrov. Regularity of conjugacies between critical circle maps: an experimental study. Experiment. Math., 11(2):219-241, 2002.

[9] Rafael de la Llave and Arturo Olvera. The obstruction criterion for non-existence of invariant circles and renormalization. Nonlinearity, 19(8):1907-1937, 2006.

[10] S. J. Shenker and L. P. Kadanoff. Critical behavior of a KAM surface. I. Empirical results. J. Statist. Phys., 27(4):631-656, 1982.

[11] Andreas Stirnemann. Renormalization for golden circles. Comm. Math. Phys., 152(2):369-431, 1993.

[12] Andreas Stirnemann. Towards an existence proof of MacKay's fixed point. Comm. Math. Phys., 188(3):723-735, 1997.

[13] N. Metropolis, M. L. Stein, and P. R. Stein. On finite limit sets for transformations on the unit interval. J. Combinatorial Theory Ser. A, 15:25-44, 1973.

[14] B. Derrida, A. Gervois, and Y. Pomeau. Universal metric properties of bifurcations of endomorphisms. J. Phys. A, 12(3):269-296, 1979.

[15] John Milnor and William Thurston. On iterated maps of the interval. In Dynamical Systems (College Park, MD, 1986-87), volume 1342 of Lecture Notes in Math., pages 465-563. Springer, Berlin, 1988.

[16] N. S. Manton and M. Nauenberg. Universal scaling behaviour for iterated maps in the complex plane. Comm. Math. Phys., 89(4):555-570, 1983.

[17] Michael Widom. Renormalization group analysis of quasiperiodicity in analytic maps. Comm. Math. Phys., 92(1):121-136, 1983.

[18] Rafael de la Llave, Arturo Olvera, and Nikola Petrov. in progress.

[19] M.-R. Herman. Sur la conjugaison différentiable des difféomorphismes du cercle à des rotations. Inst. Hautes Études Sci. Publ. Math., 49:5-233, 1979.

[20] Ya. G. Sinal̆ and K. M. Khanin. Smoothness of conjugacies of diffeomorphisms of the circle with rotations. Uspekhi Mat. Nauk, 44(1(265)):57-82, 247, 1989.

[21] J.-P. Eckmann, H. Epstein, and P. Wittwer. Fixed points of Feigenbaum's type for the equation $f^{p}(\lambda x) \equiv \lambda f(x)$. Comm. Math. Phys., 93(4):495-516, 1984.

[22] P. Collet, J.-P. Eckmann, and O. E. Lanford, III. Universal properties of maps on an interval. Comm. Math. Phys., 76(3):211-254, 1980.

[23] R. S. MacKay and I. C. Percival. Universal small-scale structure near the boundary of Siegel disks of arbitrary rotation number. Phys. D, 26(1-3):193-202, 1987.

[24] Andreas Stirnemann. Existence of the Siegel disc renormalization fixed point. Nonlinearity, 7(3):959-974, 1994.

[25] C. T. McMullen. Self-similarity of Siegel disks and Hausdorff dimension of Julia sets. Acta Math., 180(2):247-292, 1998.

[26] D. Gaidashev and M. Yampolsky. Preprint http://arxiv.org/abs/math/0603008, 2006.

[27] C. T. McMullen. Complex Dynamics and Renormalization. Princeton University Press, Princeton, NJ, 1994.

[28] C. T. McMullen. Renormalization and 3-Manifolds Which Fiber over the Circle. Princeton University Press, Princeton, NJ, 1996.

[29] O. E. Lanford, III. Renormalization group methods for circle mappings. In Statistical Mechanics 
and Field Theory: Mathematical Aspects (Groningen, 1985), pages 176-189. Springer-Verlag, Berlin, 1986.

[30] O. E. Lanford, III. Renormalization group methods for circle mappings. In Nonlinear Evolution and Chaotic Phenomena (Noto, 1987), pages 25-36. Plenum, New York, 1988. 